\title{
A reporting tool for practice guidelines in healthcare: the RIGHT Statement
}

Yaolong Chen, Kehu Yang*, Ana Marušić, Amir Qaseem, Joerg J Meerpohl, Signe Flottorp, Elie A Akl, Holger J Schünemann, Edwin SY Chan, Yngve Falck-Ytter, Faruque Ahmed, Sarah Barber, Chiehfeng Chen, Mingming Zhang, Bin Xu, Jinhui Tian, Fujian Song, Hongcai Shang, Kun Tang, Qi Wang, Susan L Norris* for RIGHT Working Group

Evidence-Based Medicine Center, School of Basic Medical Sciences, Lanzhou University, Lanzhou, China (Y Chen PhD, K Yang MMed, J Tian PhD, Qi Wang, MMed);

Key Laboratory of Evidence Based Medicine and Knowledge Translation of Gansu Province, Lanzhou, China (Y Chen PhD, Yang MMed, J Tian PhD);

Key Laboratory of Chinese Internal Medicine of Ministry of Education, Dongzhimen Hospital, Beijing University of Chinese Medicine. China (Y Chen PhD, H Shang MD, PhD)

Department of Research in Biomedicine and Health, and Cochrane Croatia, School of Medicine, University of Split, Soltanska, Croatia. (Ana Marušić MD, $\mathrm{PhD}$ )

American College of Physicians, Philadelphia, USA. (A Qaseem MD, PhD, MHA)

Centre de Recherche Épidémiologie et Statistique Sorbonne Paris Cité - U1153, Inserm / Université Paris Descartes, Cochrane France, Hôpital Hôtel-Dieu, Paris, France (JJ Meerpohl MD)

Cochrane Germany, Medical Center - University of Freiburg, Freiburg, Germany (JJ Meerpohl MD)

Department for evidence synthesis, the Norwegian Institute of Public Health, Oslo, Norway (S Flottorp MD, PhD)

Department of Internal Medicine, American University of Beirut, Beirut, Lebanon (EA Akl, MD, $\mathrm{MPH}, \mathrm{PhD}$ )

Department of Clinical Epidemiology and Biostatistics, McMaster University, Hamilton, Canada (HJ Schünemann MD, PhD, EA Akl MD, MPH, PhD)

Cochrane Singapore, Singapore Clinical Research Institute, Duke-NUS Medical School, Singapore (E Chan PhD)

Department of Medicine, Case and VA Medical Center, Case Western Reserve University, Cleveland, USA (Y Falck-Ytter MD)

Centers for Disease Control and Prevention, Atlanta, USA (F Ahmed PhD)

South Africa WHO Country Office, Pretoria, South Africa (S Barber PhD)

Division of Plastic Surgery, Department of Surgery, Wan Fang Hospital, Department of Public Health, School of Medicine, College of Medicine, Cochrane Taiwan, Taipei Medical University, Taiwan (C Chen MD, MPH, PhD)

Chinese Cochrane Centre, West China Hospital, Sichuan University, Chengdu, China (M Zhang M.Sc)

Nanjing University of Chinese Medicine, Nanjing, China (B Xu MD)

Norwich Medical School, Faculty of Medicine and Health Science, University of East Anglia, Norwich, UK (F Song PhD)

Department of Global Health, Peking University, Beijing, China (K Tang PhD)

Guidelines Review Committee Secretariat, World Health Organization, Geneva, Switzerland (SL

Norris MD, MPH, MSc)

* corresponding authors 


\section{$44 \quad$ Abstract}

The quality of reporting of practice guidelines is often poor and there is no widely accepted guidance or standards for the reporting of practice guidelines in healthcare. An international working group (the RIGHT working group) was therefore established to address this gap. The group followed an existing framework for developing health research reporting guidelines and the EQUATOR (Enhancing the QUAlity and Transparency Of health Research) Network approach.

We developed a checklist and an explanation and elaboration document. The RIGHT checklist includes 22 items that we consider essential for good reporting of practice guidelines. These items encompass basic information (items 1-4), background (items 5-9), evidence (items 10-12), recommendations (items 13-15), review and quality assurance (items 16-17), funding and declaration and management of interests (items 18-19), and other information (items 20-22). The RIGHT checklist can assist developers when reporting their guidelines, support journal editors and peer reviewers when considering guideline reports, and help healthcare practitioners understand and implement a guideline.

Primary Funding Source: National Natural Science Foundation of China, Grant No. 81503459 


\section{Introduction}

Clear, explicit and transparent practice guidelines enable healthcare practitioners, health administrators, program managers, and the public to understand and implement recommendations that may positively impact patients and populations (1). However, the quality of reporting of practice guidelines appears to be low (2) and current tools to address this are either outdated, have a narrow focus, or combine reporting and quality assessment in a single instrument. The Conference on Guideline Standardization (COGS) published a checklist for reporting of clinical practice guidelines (last updated in 2003) which focuses mainly on clinical medicine, and thus it may not be directly applicable to public health or to other types of guidelines (3). The AGREE instrument was developed for both quality assessment and reporting, although it is widely regarded as an evaluation tool $(6,7)$. Multi-function tools may not be optimal as it is important to distinguish between tools that address reporting and those that assess methodological quality as they differ in purpose, structure and content (8). Recently the AGREE Next Steps Consortium published the AGREE Reporting Checklist based on the AGREE instrument $(4,5)$, however it is limited to items derived from the original tool, was developed by a small group of researchers and does not provide detailed explanation or guidance as to how to use the tool.

\section{Development of the RIGHT Checklist}

A multidisciplinary international team that included policy makers, methodologists, epidemiologists, clinicians, editors and consumer representatives from 12 countries across Asia, Africa, Europe, Oceania and North America was established in 2013. It aimed to develop a tool focusing on the essential items for reporting of guidelines-RIGHT (Reporting Items for Practice Guidelines in HealThcare). Development of the RIGHT checklist followed the framework for developing health research reporting guidelines (9). We registered the project in the EQUATOR (Enhancing the QUAlity and Transparency Of health Research) library (10). We established two groups: the RIGHT development group and the Delphi panelists group. The RIGHT development group drafted the project proposal, generated suggested items, recruited Delphi panelists, designed the questionnaires for the Delphi survey, and drafted the final report. The Delphi panelists group reviewed the proposal, participated in three rounds of Delphi surveys, came to consensus on the items included in the final checklist, and reviewed the final manuscript.

The RIGHT development group implemented a four-step approach to generate potential items for the checklist. First, the group reviewed ten representative reporting guidelines highlighted in the EQUATOR library to determine how they generated potential items (11). The ten documents encompassed a wide variety of reporting tools, including for randomized controlled trials, diagnostic studies, observational studies, animal research, economic evaluation and systematic reviews. One tool generated items based on a systematic review (12) while the others used surveys, group meetings, literature reviews, or combined approaches (13-21). Second, we conducted a comprehensive search of handbooks and other documents to identify standards or tools for guideline reporting (see Appendix 1). Third, two sub-groups from the RIGHT development group, each composed of two experienced investigators, independently extracted potential checklist items from all documents identified in the first two steps. Last, the RIGHT development group held a face-toface meeting to aggregate all potential items and remove duplicates. After further discussion, 48 items were included in the initial list of potential items. Readers can obtain the search results and 
initial list of items from the RIGHT website (22).

For the Delphi method, we recruited 17 individuals with experience in the development of practice guidelines or reporting guidelines. These individuals encompassed a broad range of disciplines as well as diverse geographic representation. The Delphi technique followed the recommendations proposed by Murphy and Sinha $(23,24)$ and included three rounds of email-based surveys. Panelists rated each item on a scale of 1 (not important) to 5 (very important), suggested new items, and provided comments that were circulated in subsequent rounds. All panelists were asked to disclose any conflicts of interest before beginning the Delphi survey. The response rate was $100 \%$ for all three rounds of the Delphi process.

This study was funded by National Natural Science Foundation of China and the funder had no role in study design, data collection and analysis, decision to publish, or preparation of the manuscript.

\section{RIGHT Checklist}

The RIGHT checklist consists of 22 items that we consider essential for good reporting of practice guidelines (Table). These items encompass the following domains: basic information (item 1-4), background (items 5-9), evidence (items 10-12), recommendations (items 13-15), review and quality assurance (items 16-17), funding, declaration and management of interests (items 18-19), and other information (items 20-22).

\section{Discussion}

The RIGHT checklist can assist guideline developers when reporting their guidelines, support journal editors and peer reviewers when considering guideline reports, and help healthcare practitioners understand and implement a guideline. The checklist is useful for clinical practice guidelines as well as guidelines in public health and other healthcare fields since users and evaluators need a clear, explicit description of the processes and procedures used to develop a guideline, and access to the evidence used to formulate each recommendation.

The RIGHT checklist does not prescribe a specific format for the reporting of guidelines. Rather, each checklist item should be clearly presented and in sufficient detail somewhere in the guideline. Order and format for each item depend on the developers' preferences, style of the publication, and most importantly, the end-users' needs. We recommend against deriving a score from the RIGHT Checklist: the items may not be equally weighted and scores have been demonstrated to be problematic in research synthesis $(25,26)$.

We emphasize that the RIGHT checklist was not developed as a tool for assessing the quality of published practice guidelines; such instruments exist elsewhere including AGREE (27) and others (28). Rather, RIGHT is intended to complement these existing tools. RIGHT was also not developed as guidance for developing guidelines. Many handbooks exist for this purpose, along with the GIN-McMaster Guideline development checklist - a practical tool for guideline development supported by learning resources (29). Readers should carefully select a tool according 
to their specific needs.

The RIGHT checklist differs from the new AGREE reporting checklist (5) in several important ways. First, the structure of the AGREE reporting checklist follows the domains of AGREE II: scope and purpose, stakeholder involvement, rigour of development, clarity of presentation, applicability, and editorial independence. In contrast, the RIGHT checklist emulates the approach used by other reporting guidance statements such as CONSORT(15) and PRISMA(13), ordering items as the developer and reader would encounter them. Thus RIGHT starts with the title, then the executive summary, for example. Second, RIGHT includes important items that should be reported in a guideline that were not included in the AGREE reporting checklist: quality assurance, access, suggestions for further research, and limitation of the guideline. RIGHT highlights the importance of reporting PICO questions and quality of the body of evidence, and includes seven sub-items on the formulation of recommendations from evidence. Finally, the RIGHT explanation and elaboration statement (appendix 2) provides detailed information and examples, which are not part of the AGREE reporting checklist.

\section{Implementation}

Endorsement and implementation of reporting guidelines may help reduce wasteful research and increase the potential impact of research on health (30). We plan to use a number of approaches to promote implementation of the RIGHT checklist: ask authors of international guideline handbooks to add the RIGHT checklist into new versions of their handbooks; contact the editors of the core clinical journals in MEDLINE (https://www.nlm.nih.gov/bsd/aim.html) to elicit their support and encourage them to endorse the RIGHT checklist; and inform guideline developers at international and national agencies, as well as professional societies about RIGHT.

\section{Strengths and limitations}

We followed an explicit, transparent and documented process for developing the RIGHT checklist and we provide an accompanying explanation and elaboration statement (See online appendix). Individuals from key international organizations and institutions that focus on development and implementation of guidelines contributed to this work, including the EQUATOR network, Guidelines International Network, the GRADE Working Group, the AGREE Collaboration and the Cochrane Collaboration. The draft checklist and explanation and elaboration statement underwent extensive peer review by experts in guideline development with a variety of perspectives. It is possible that we missed important items when we developed our initial list of items, however we made every effort to minimize this possibility by examining a large number of guidance documents and manuals produced by guideline developers and by consulting a broad range of experts in this field.

\section{Future development and research}

The RIGHT checklist is currently available in English, German, Croatian, Japanese, Korean, Simplified and Traditional Chinese, and we encourage groups to undertake additional translations. We plan to develop RIGHT extensions, including RIGHT-P (for Guideline Proposals), RIGHT-COI (for Conflicts of Interest), and RIGHT-A (for Acupuncture). We ask those who aim to develop related standards or perform translations to contact the corresponding author of this paper to 
coordinate efforts and to avoid duplication.

As for any other reporting standard, the RIGHT checklist is an evolving document that needs continual assessment, improvement, and updating. We will revise the checklist in the future, taking into account user feedback, results of formal and informal evaluations, and new studies on guideline reporting methods. We encourage users to submit their comments via the RIGHT website.

Acknowledgements: We are grateful to the individuals who responded to the Delphi survey and for their thoughtful comments.

\section{Contributors}

YC, SLN and KY conceived of RIGHT project and drafted the project proposal. AM, AQ, JM, SF, EA, EC, YFY, FA, SR, CC, MZ, BX were Delphi panelists and gave comments and suggestions on the draft item list. YC, KY, FS and KT generated suggested items, designed the questionnaires for the Delphi survey and did the statistical analysis. YC and SLN drafted the manuscript and all authors critically reviewed and revised it for important intellectual content. YC is the guarantor of the manuscript, and affirms that the manuscript is an honest, accurate, and transparent account of the study being reported. All authors approved the final version of this article.

\section{Disclaimer}

The findings and conclusions in this article are those of the authors and do not necessarily represent the views of WHO or the US Centers for Disease Control and Prevention.

Competing interests: All authors have completed the ICMJE uniform disclosure form at www.icmje.org/coi_disclosure.pdf (available on request from the corresponding author) and declare no financial relationships with any organisations that might have an interest in the submitted work in the previous three years. A number of the authors are active members of the GRADE Working Group (YC, JM, SF, EA, HJS, YFY, FA, SLN). YC is the member of the AGREEHealth System team.

Ethics approval: Not needed.

Funding: This study was funded by National Natural Science Foundation of China, Grant No. 81503459; China Fundamental Research Funds for the Central Universities, Grant No. 2016LZUJBZX159; The Open Fund of the Key Laboratory of Evidence-Based Medicine and Knowledge Translation of Gansu Province, Grant No. EBM1305.

\section{References}

1. Oxman AD, Fretheim A, Schünemann HJ. Improving the use of research evidence in guideline development: 14. Reporting guidelines. Health Res Policy Syst. 2006;4:26. 
2. Grilli R, Magrini N, Penna A, Mura G, Liberati A. Practice guidelines developed by specialty societies: the need for a critical appraisal. Lancet. 2000;355(9198):103-6.

3. Shiffman RN, Shekelle P, Overhage JM, Slutsky J, Grimshaw J, Deshpande AM. Standardized reporting of clinical practice guidelines: a proposal from the Conference on Guideline Standardization. Ann Intern Med. 2003;139(6):493-8.

4. Brouwers MC, Kho ME, Browman GP, Burgers JS, Cluzeau F, Feder G, et al. AGREE II: advancing guideline development, reporting and evaluation in health care. CMAJ. 2010;182(18):E839-42.

5. Brouwers MC, Kerkvliet K, Spithoff K, Consortium ANS. The AGREE Reporting Checklist: a tool to improve reporting of clinical practice guidelines. BMJ. 2016;352:i1152.

6. Oxman AD, Schünemann HJ, Fretheim A. Improving the use of research evidence in guideline development: 16. Evaluation. Health Res Policy Syst. 2006;4:28.

7. Wilson KC, Irwin RS, File TM, Schünemann HJ, Guyatt GH, Rabe KF. Reporting and publishing guidelines: article 12 in Integrating and coordinating efforts in COPD guideline development. An official ATS/ERS workshop report. Proc Am Thorac Soc. 2012;9(5):293-7.

8. Huwiler-Muntener K, Juni P, Junker C, Egger M. Quality of reporting of randomized trials as a measure of methodologic quality. JAMA. 2002;287(21):2801-4.

9. Moher D, Schulz KF, Simera I, Altman DG. Guidance for developers of health research reporting guidelines. PLoS Med. 2010;7(2):e1000217.

10. Reporting Items for Guidelines in Health Systems. [Internet]. Minervation Ltd. [cited 2015 Jul 9]. Available from: http://www.equator-network.org/library/reporting-guidelines-under-development.

11. Reporting guidelines for main study types. [Internet]. Minervation Ltd. [cited 2015 Jul 9]. Available from: http://www.equator-network.org/library/.

12. Chan AW, Tetzlaff JM, Altman DG, Laupacis A, Gotzsche PC, Krleza-Jeric K, et al. SPIRIT 2013 statement: defining standard protocol items for clinical trials. Ann Intern Med. 2013;158(3):200-7.

13. Moher D, Liberati A, Tetzlaff J, Altman DG. Preferred reporting items for systematic reviews and meta-analyses: the PRISMA statement. BMJ. 2009;339:b2535.

14. Gagnier JJ, Kienle G, Altman DG, Moher D, Sox H, Riley D. The CARE guidelines: consensusbased clinical case report guideline development. J Clin Epidemiol. 2014;67(1):46-51.

15. Schulz KF, Altman DG, Moher D. CONSORT 2010 statement: updated guidelines for reporting parallel group randomised trials. BMJ. 2010;340:c332.

16. von Elm E, Altman DG, Egger M, Pocock SJ, Gotzsche PC, Vandenbroucke JP. Strengthening the Reporting of Observational Studies in Epidemiology (STROBE) statement: guidelines for reporting observational studies. BMJ. 2007;335(7624):806-8.

17. Tong A, Sainsbury P, Craig J. Consolidated criteria for reporting qualitative research (COREQ): a 32-item checklist for interviews and focus groups. Int J Qual Health Care. 2007;19(6):349-57.

18. Bossuyt PM, Reitsma JB, Bruns DE, Gatsonis CA, Glasziou PP, Irwig LM, et al. Towards complete and accurate reporting of studies of diagnostic accuracy: the STARD initiative. BMJ. 2003;326(7379):41-4.

19. Davidoff F, Batalden P, Stevens D, Ogrinc G, Mooney SE. Publication guidelines for quality improvement studies in health care: evolution of the SQUIRE project. BMJ. 2009;338:a3152.

20. Husereau D, Drummond M, Petrou S, Carswell C, Moher D, Greenberg D, et al. Consolidated Health Economic Evaluation Reporting Standards (CHEERS) statement. BMJ. 2013;346:f1049.

21. Kilkenny C, Browne WJ, Cuthill IC, Emerson M, Altman DG. Improving bioscience research 
reporting: The ARRIVE guidelines for reporting animal research. J Pharmacol Pharmacother. 2010;1(2):94-9.

22. The RIGHT Statement. [Internet]. [cited 2015 Jul 9]. Available from: http://www.rightstatement.org/.

23. Murphy MK, Black NA, Lamping DL, McKee CM, Sanderson CF, Askham J, et al. Consensus development methods, and their use in clinical guideline development. Health Technol Assess. 1998;2(3):i-iv, 1-88.

24. Sinha IP, Smyth RL, Williamson PR. Using the Delphi technique to determine which outcomes to measure in clinical trials: recommendations for the future based on a systematic review of existing studies. PLoS Med. 2011;8(1):e1000393.

25. Greenland S, O'Rourke K. On the bias produced by quality scores in meta-analysis, and a hierarchical view of proposed solutions. Biostatistics. 2001;2(4):463-71.

26. Juni P, Witschi A, Bloch R, Egger M. The hazards of scoring the quality of clinical trials for metaanalysis. JAMA. 1999;282(11):1054-60.

27. Brouwers MC, Kho ME, Browman GP, Burgers JS, Cluzeau F, Feder G, et al. The Global Rating Scale complements the AGREE II in advancing the quality of practice guidelines. J Clin Epidemiol. 2012;65(5):526-34.

28. Grimmer K, Dizon JM, Milanese S, King E, Beaton K, Thorpe O, et al. Efficient clinical evaluation of guideline quality: development and testing of a new tool. BMC Med Res Methodol. 2014;14:63.

29. Schünemann HJ, Wiercioch W, Etxeandia I, Falavigna M, Santesso N, Mustafa R, et al. Guidelines 2.0: systematic development of a comprehensive checklist for a successful guideline enterprise. CMAJ. 2014;186(3):E123-42.

30. Glasziou P, Altman DG, Bossuyt P, Boutron I, Clarke M, Julious S, et al. Reducing waste from incomplete or unusable reports of biomedical research. Lancet. 2014;383(9913):267-76. 